\title{
NUEVAS COMBINACIONES Y UNA NUEVA VARIEDAD DE LYCIUM (SOLANACEAE) DE NORTEAMERICA*
}

\section{FERNANDO CHIANG**}

\section{RESUMEN}

Se proponen 3 combinaciones nomenclaturales para Lycium de Norteamérica: $L$. macrodon var. dispermum, $L$. nodosum var. isthmense, L. parishii var. modestum, L. puberulum var. berberidoides, y una nueva variedad: L. californicum var. interior.

\section{ABSTRACT}

Three name combinations for North American Lycium: L. macrodon var. dispermum, L. nodosum var. istbmense, L. parisbii var. modestum, L. puberulum var. berberidoides, and a new variety: L. californicum var. interior, are proposed.

Con el fin de formalizar los nombres de algunos de los taxa cuyos números cromosómicos se presentan en otro artículo del mismo número de esta revista, con base en un estudio reciente sobre la taxonomía de las especies norteamericanas de Lycium (Chiang, 1981), se proponen las siguientes combinaciones:

Lycium macrodon var. dispermum (Wiggins) Chiang, stat. et comb. nov.

L. dispermum Wiggins, Contr. Dudley Herb. 1:204. 1934. Tipo: México: Sonora: ribera norte del Río Yaqui, en Tacimaroa, vado entre Corral y Ciudad Obregón (Cajeme), 2 de marzo de 1953, Wiggins 6373 (holótipo: DS; isótipo: US).

Esta variedad se distingue de la var. macrodon por su corola pubescente, lóbulos corolinos más densamente ciliolados, estambres y estilo más exertos, y por las hojas glabras. Las características anteriores son mencionadas por Wiggins (1934) al discutir la relación de $L$. dispermum con $L$. macrodon. También mencionó la adherencia de los filamentos al tubo corolino a una distancia de $2 / 5$ de la longitud del tubo en $L$. dispermum, en contraste con la adherencia de los filamentos cerca de la mitad de la

* Trabajo aceptado para su publicación en Nioviembre de 1982.

** Departamento de Botánica, Instituto de Biología, UNAM. Apartado Postal 70-233, Del. Coyoacán, 04510 México, D. F. 
longitud del tubo en $L$. macrodon. El punto de adherencia de los filamentos usado por Wiggins es parte de la variación encontrada en $L$. macrodon, de manera que este carácter pierde importancia. Las únicas diferencias significativas entre $L$. dispermum y L. macrodon son la pubescencia foliar, la densidad ciliolar en los lóbulos corolinos y el grado en que sobresalen los estambres y el estilo. Tomando en cuenta que las hojas, flores y frutos son del mismo tipo en ambos taxa, y por la similitud de los patrones cromatográficos en papel, es que se propone la transferencia de $L$. dispermum a entidad varietal de $L$. macrodon.

Lycium nodosum var. istbmense (Chiang) Chiang, stat. et comb. nov.

L. isthmense Chiang, Bol. Soc. Bot. México 35: 3-5. 1975.

Tipo: México: Oaxaca: cerca de la orilla de la laguna interior en la Playa La Ventosa, aproximadamente $7 \mathrm{~km}$ al Este de Salina Cruz, 19 de junio de 1974, Wendt \& Chiang 315 (holótipo: LL; isótipo: MEXU).

Esta variedad encuentra su pariente más próximo en $L$. nodosum Miers var. nodosum, de Argentina, del que difiere en tener pecíolos más delicados y largos, teniendo prácticamente los mismos caracteres florales.

Lycium parishii var. modestum (I. M. Johnston) Chiang, stat. et comb. nov.

L. modestum I. M. Johnston, J. Arnold Arbor. 21: 73. 1940.

Tipo: México: San Luis Potosí: $13 \mathrm{~km}$ al NW de Cedral, 12 de septiembre de 1938,Johnston 7605 (holótipo: GH; isótipo: US).

Esta variedad se distingue de la var. parisbii por la escasa pubescencia glandular del exterior de la corola y por la baja estatura de los arbustos, que no pasan de $1.5 \mathrm{~m}$. Se distribuye en planicies aluviales, llanos gipsíferos y laderas calizas en el centro y sur de Coahuila, oeste de Nuevo León y norte de San Luis Potosí, completamente aislada de la var. parisbii, un taxon del Desierto Sonorense. Johnston (1940) describió el hábito de esta variedad como un arbusto bajo, deprimido con tallos enraizantes extendidos ("depressed low bush with spreading root stems"), enfatizando en particular, el hábito deprimido. Sin embargo, en la vecindad de la localidad tipo, se encuentran plantas de esta variedad robustas y erectas.

L. puberulum var. berberidoides (Correll) Chiang, stat. et comb. nov.

L. berberidoides Correll, Wrightia 3(7): 138. 1965, "berberioides"* .

Tipo: E.U.A.: Texas: Brewster Co.: $4.8 \mathrm{~km}$ al sur de Persimmon Gap, Big Bend National Park, 15 de junio de 1964, Correll, Gentry \& Hanson 29712 (holótipo: LL; isótipos: MEXU, TEX; y GH, US, fide Correll). dice:

Correll (1965), al discutir las relaciones de su nueva especie (L. berberidoides)

It is unquestionably related to $L$. puberulum A. Gray. However, that species has its non-glaucous leaves, pedicels and calyx densely covered with a glandular

* Aunque el epíteto específico se publicó como berberioides, filológicamente es preferible berberidoides y debe ser usado (Art*c. 32.4, International Code of Botanical Nomenclature, 1978). 
puberulence. The triangular-ovate calyx-lobes are also erect or only slightly spreading and eventually they clasp the fruit. The corolla-lobes, too, in that species are also only spreading and not reflexed.

La única diferencia real se encuentra en la pubescencia; los lóbulos de la corola pueden ser, ya patentes, ya reflexos, en $L$. puberulum var. puberulum. Los lóbulos del cáliz observados en el material tipo de $L$. berberidoides son patentes o erectos, y no "usually reflexed", como se menciona en la descripción original de $L$. berberidoides. Correll describió el fruto de L. berberidoides como "fruit (immature) glaucous". El ejemplar Whitehouse 19679 (ARIZ), claramente var. berberidoides, de la misma área general que el ejemplar tipo, presenta frutos maduros del mismo tipo que los de la var. puberulum. Los caracteres florales son idénticos en los dos taxa, y sus patrones cromatográficos en papel son muy similares también.

Lycium californicum var. interior Chiang, var. nov., frutex prostratus fasciculis dense foliosis, folia semiteretia interdum subrevoluta $4-12 \mathrm{~mm}$ longa; flores $4-9 \mathrm{~mm}$ longis. Tipo: México: Coahuila: Llano del Guaje, márgenes de playa entre Lomas del Aparejo y Tanque La India, cerca de lat. $28^{\circ} 00^{\prime}-28^{\circ} 05^{\prime} \mathrm{N}, 28$ de agosto de 1940, Johnston \& Muller 780 (holótipo: LL; isótipo: GH).

Arbusto postrado, con fascículos densamente hojosos, hojas semiteretes, en ocasiones subrevolutas, 4-12 mm de largo; flores 4-9 mm de largo.

Distribución: llanos salinos aluviales o cerca de la orilla de lagunas endorreicas, en el Oeste de Coahuila y en área donde confluyen los estados de Coahuila, Zacatecas y San Luis Potosí.

Período de floración: junio a agosto.

Ejemplares adicionales examinados: COAHUILA: Llano del Guaje, orillas de playa entre Lomas del Aparejo y Tanque La India, cerca de lat. $28^{\circ}-28^{\circ} 05^{\prime} \mathrm{N}, 28$ ago. 1940, Johnston \& Muller 780 ( $\mathrm{GH}, \mathrm{LL}$ ); camino de Puerto del Caballo hacia el Este, $16 \mathrm{~km}$ a través del amplio valle a las lomas en Tanque Jericó, cerca de lat. 27034' N, 22-23 ago. 1941, Johnston 8236 (GH, LL); Laguna de Leche (orilla NE), 28 ago. 1941, Johnston 8593 (GH, LL); Llano de Guaje, cerca del Tanque de La India, aprox. 28 $00^{\prime}$ N, 25 ago. 1941, Stewart 1163 (GH, LL); $7.4 \mathrm{~km}$ al SE de Encarnación de Guzmán, sobre el camino a la Colonia de San Sebastián, $5.5 \mathrm{~km}$ al NW del Rancho San José de Guzmán, en Potrero de San Isidro, 2848’ N, 10059’ W, 20 ago. 1974, Wendt \& Lott 601 (LL); $12.2 \mathrm{~km}$ al SE de Encarnación de Guzmán, sobre el camino a La Colonia de San Sebastián, $0.6 \mathrm{~km}$ al NW del Rancho San José

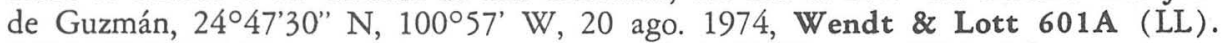
SAN LUIS POTOSI: $27.4 \mathrm{~km}$ al NW de Cedral, 11-12 sept. 1938, Johnston 7610 (GH). ZACATECAS: $8.5 \mathrm{~km}$ al SE de Puerto de Rocamontes, sobre la Carret. 54, $24^{\circ} 42^{\prime} \mathrm{N}, 101^{\circ} 15^{\prime} \mathrm{W}, 18$ jun. 1972, Chiang et al. 7941 (LL); $1 \mathrm{~km}$ al W de Guadalupe Garcerón sobre la carretera a Concepción del Oro, 3 jul. 1973, Johnston et al. 11591 (LL).

\section{AGRADECIMIENTOS}

Se agradece al Dr. T. P. Ramamoorthy sus sugerencias en la presentación de este artículo. 
BOLETIN DE LA SOCIEDAD BOTANICA DE MEXICO No. 43, 1982

\section{BIBLIOGRAFIA}

CORRELL, D. S. 1965. Some additions and corrections to the Flora of Texas. Wrightia 3(7): 126-140 (Lycium, págs. 138-139).

CHIANG, F. 1981. A Taxonomic Study of the North American Species of Lycium (Solanaceae) Ph. D. Dissertation. The University of Texas at Austin. 287 p.

JOHNSTON, I. M. 1940. New phanerogams from Mexico. II. J. Arnold Arbor. 21 : 67-75 (Lycium, págs. 72-74).

WIGGINS, I. L. 1934. A report on several species of Lycium from the southwestern deserts. Contr. Dudley Herb. 1(6): 197-209. 